\title{
Reforma laboral en México, ¿Solución a la justicia social? Labor reform in Mexico, Solution to social justice?
}

\author{
María del Rocío García Sánchez \\ Universidad Autónoma de Guerrero, México \\ mrociogsz@yahoo.com.mx \\ Joaquín Reyes Añorve \\ Universidad Autónoma de Guerrero, México \\ joaquin.reyes94@yahoo.com.mx \\ Timoteo Antúnez Salgado \\ Universidad Autónoma de Guerrero, México \\ timoteoantunez@yahoo.com \\ Guadalupe Godínez Alarcón \\ Universidad Autónoma de Guerrero, México \\ cenself12@hotmail.com
}

Alejandro Silva González

Universidad Autónoma de Guerrero, México

dr.asg@hotmail.com

\section{Resumen}

La crisis de la economía mundial desde la década de los años setenta hizo necesario hacer reformas estructurales a nivel global. Los países latinoamericanos, en la década de los años noventa, idearon una serie de reformas estructurales a fin de lograr un alto rendimiento en el sector laboral y frenar de esta manera los costos del despido. Se consideró que el sistema universal de protección social en la mayoría de los países de la región, que tradicionalmente habían regido la actividad laboral, no cumplía con las expectativas de desarrollo económico global pues no demostró un aumento de empleos y de inversión extranjera; sin embargo, las reformas instauradas en la región latinoamericana tampoco han cumplido con el objetivo de generar trabajo; por el contrario, sigue habiendo un evidente desempleo y una mayor 
desprotección social, lo que entorpece el ejercicio de otros derechos y crea un factor poderoso de discriminación laboral.

Palabras clave: reforma laboral, justicia social y desempleo.

\section{Abstract}

The crisis of the world economy since the 1970s made it necessary to make structural reforms at the global level. Latin American countries, in the Decade of the 1990s, devised a series of structural reforms in order to achieve high performance in the labor sector and curb this way the dismissal costs. Is considered that the system universal of protection social in it most of them countries of the region, that traditionally had governed the work activity, does not met the expectations of global economic development because did not showed an increase of jobs and of foreign investment; however, the reforms established in the region Latin American nor have met with the objective of generating work; conversely, there is still an evident unemployment and a greater lack of social protection, which hinders the exercise of other rights and creates a powerful factor of employment discrimination.

Key words: labor reform, social justice and unemployment.

Fecha Recepción: Marzo $2015 \quad$ Fecha Aceptación: Octubre 2015

\section{Introducción}

En México, el 30 de noviembre de 2012 se publicó en el Diario Oficial de la Federación la primera reforma sustancial a la Ley Federal del Trabajo desde su promulgación en 1970. Un propósito de la reforma fue la necesidad de actualizar la ley laboral permitiendo oficialmente empleos de unas cuantas horas y no por jornadas completas, y la celebración 
de contratos a prueba, que en la opinión de expertos en materia laboral facilita el despido de trabajadores.

Estas reformas en materia laboral se concibieron bajo la visión de impulsar la productividad de las relaciones laborales y propiciar la competitividad de la economía, elementos necesarios para la inserción de la economía mexicana en el proceso de globalización. Principios como la estabilidad laboral, la protección del trabajador frente a los riesgos del desempleo, la enfermedad y la vejez fueron considerados como obstáculos demasiado caros para la economía del país, que perjudican, además, los procesos de crecimiento económico y de desarrollo.

En este sentido, el gobierno federal insistió que con la reforma a la ley laboral sería posible crear nuevos miles de empleos. Dichas reformas incluyeron esquemas como:

- Ofrecer nuevas modalidades de contratación, con lo que se pretende generar las condiciones para que un mayor número de personas puedan integrarse a puestos de trabajo de la economía formal.

-Periodos de prueba.

-Contratos de capacitación inicial.

-Contratos para el trabajo de temporada.

- Regular la subcontratación de personal u outsourcing.

- Brindar seguridad jurídica a los mexicanos que van a laborar en el extranjero.

- Reconocer el teletrabajo o el trabajo a distancia haciendo uso de las tecnologías de información.

- Incorporar la definición de trabajo decente que promueve la Organización Internacional del Trabajo (OIT), la cual está acorde con la definición de trabajo digno establecido en la constitución.

- Propiciar mejores condiciones de trabajo a grupos vulnerables como son: los trabajadores eventuales y del campo, los domésticos y los mineros.

○ Instaurar un procedimiento ágil y abreviado para negociar conflictos originados derivados de la seguridad social.

- Considerar una nueva causal de rescisión de relación laboral sin perjuicio para el trabajador, cuando el patrón le exija realizar actos o conductas o tener comportamientos que menoscaben o atenten contra su dignidad. 
- Fortalecer los derechos de las trabajadoras y propiciar la equidad al instituir la figura de licencias por maternidad.

- Establecer que los sueldos caídos del trabajador ganados en un juicio laboral por despido injustificado ante la Junta de Conciliación y Arbitraje se limitarán a un año y $2 \%$ a razón de 15 meses del salario del trabajador (antes de la reforma no había un límite para el pago de dichas percepciones).

- Mejorar las condiciones de trabajo de los empleados domésticos.

- Actualizar las tablas de enfermedades de trabajo y la valoración de las incapacidades permanentes.

Aun cuando la reforma pretende mantener la seguridad social como un elemento importante en las nuevas relaciones laborales, en un Estado social una transformación de esta naturaleza debe centrarse en resolver problemas de desigualdad e injusticia social. En estos momentos, a nivel mundial estamos experimentando una severa crisis económica cuyos efectos todavía no es posible prever por completo.

\section{Presentación}

Muchos ciudadanos pese al terrible desempleo en el país se esfuerzan cada día por mejorar y elevar sus niveles de vida, sin embargo, la crisis económica que vivimos a nivel global, la pobreza, la falta de oportunidades y el despido en la mayoría de los países latinoamericanos son el principal motivo de conflicto individual.

Por ello adquiere relevancia la justicia social como el medio para lograr el reparto equitativo de los bienes sociales, que hagan posible que las clases sociales más desprotegidas cuenten con oportunidades de desarrollo. La justicia social debe ser, además, una obligación de todos los países para compensar las desigualdades que surgen en la sociedad, como un propósito para fortalecer los servicios y garantizar aquellos derechos considerados esenciales para que los individuos puedan mantener el nivel de vida requerido y ser un miembro pleno de la sociedad, como es el acceso de la salud, la alimentación, la educación pública, la vivienda, el trabajo, el salario y un sistema de pensiones que permitan alcanzar un nivel de vida digno.

La igualdad de oportunidades y el bienestar social son elementos primordiales de las sociedades que desean ser justas; son posibilidades que tiene un ser humano para ser o 
hacer lo que mejor le parezca, una forma de justicia social que ampara un sistema socialmente justo por el que las personas poseen las mismas posibilidades de acceder al bienestar social y los mismos derechos sociales. Sin embargo, la injusticia social se ha convertido en un problema mundial que ha descendido preocupantemente el bienestar económico y social de muchas personas, impidiendo el desarrollo pleno de cada individuo y condicionando la forma de vivir de quienes la padecen. Von Stein $(1949$, p. 37) ejerció una importante influencia a mediados del siglo XIX en Alemania cuando sostuvo que el Estado social era una manera concreta de evitar la revolución.

Hoy, ante la aprobación de la Declaración de Principios y Derechos Fundamentales en el Trabajo de la OIT y la instrumentación de los tratados de libre comercio, se plantea la necesidad de cumplir indudablemente con los derechos fundamentales en el trabajo con la finalidad de permitir el desarrollo de un comercio justo.

La Declaración de Principios y Derechos Fundamentales en el Trabajo de la OIT, adoptada en 1998, compromete a los Estados miembros a respetar y promover los principios y derechos comprendidos en cuatro categorías, hayan o no ratificado los convenios pertinentes. Estas categorías son:

1. La libertad de asociación y la libertad sindical y el reconocimiento efectivo del derecho de negociación colectiva.

2. La eliminación del trabajo forzoso u obligatorio.

3. La abolición del trabajo infantil.

4. La eliminación de la discriminación en materia de empleo y ocupación.

La reforma laboral en México se propuso salvaguardar el concepto de trabajo decente como principio jurídico de la normatividad laboral del Estado mexicano, la cual se lleva a cabo en un momento en el que prevalece una crisis económica internacional que ha contribuido a que el número de desempleados aumente considerablemente.

La Organización Internacional del Trabajo (OIT) indicó en su proyecto Perspectivas sociales y del empleo en el mundo. Tendencias 2016, que el mundo muestra señales de debilidad y un crecimiento económico decepcionante, lo que anticipa que durante 2016 y 
2017 habrá una desaceleración de la economía que resultará en un repunte del desempleo, escenario al que México no es ajeno.

El informe proyecta que nuestro país concluya este año con 2.4 millones de desempleados, cantidad que no disminuirá en 2017, pues para ese año prevé que se mantenga el mismo número de personas sin trabajo.

No obstante, los resultados de la Encuesta Nacional de Ocupación y Empleo (cifras durante el primer trimestre de 2015) realizada por el Instituto Nacional de Estadística y Geografía, revela que en México el subempleo va en aumento, pues las personas que buscaron un empleo adicional entre abril-junio del 2015 sumaron 4.2 millones, que equivalen al $8.3 \%$ de las personas empleadas, proporción mayor a la del mismo periodo en 2014, que fue de $8.2 \%$, es decir, se incorporaron 150000 trabajadores.

Estas categorías de trabajadores sufren generalmente altos niveles de precariedad, y dado que laboran en estas condiciones generalmente tienen un acceso limitado a los sistemas de protección social.

\section{Seguridad social y trabajo decente}

La seguridad social comprende todas las condiciones que brindan beneficios, ya sean en efectivo o en especie, para garantizar el auxilio en caso de:

a. No contar con un salario en caso de: maternidad, invalidez, enfermedad, accidentes de trabajo y enfermedades profesionales, desempleo, vejez y fallecimiento de un miembro de la familia.

b. La falta de acceso o un acceso insuficiente a la atención a la salud.

c. Un apoyo familiar insuficiente, particularmente para los hijos o adultos a cargo.

d. Pobreza y exclusión social.

El trabajo decente, por otro lado, significa que las personas durante su vida laboral aspiran a alcanzar un bienestar social para gozar una buena calidad de vida, la cual implica la oportunidad de acceder a un empleo digno que genere un ingreso justo, la seguridad en el lugar de trabajo y la protección social para las familias, mejores perspectivas de desarrollo personal e integración social, la estabilidad en el empleo y la igualdad de oportunidades para mujeres y hombres. Otra definición más de calidad de vida es la siguiente: 
Es un estado de satisfacción general, derivado de la realización de los potenciales de la persona. Posee aspectos subjetivos y aspectos objetivos. Es una sensación subjetiva de bienestar físico, psicológico y social. Incluye como aspectos subjetivos la intimidad, la expresión emocional, la seguridad percibida y la salud objetiva. Como aspectos objetivos, están el bienestar material, las relaciones armónicas con el ambiente físico y social y con la comunidad, así como la salud objetivamente percibida (Ardila, $\mathrm{R}$. 2003, en Antúnez, S. T., 2014, p.3).

El empleo productivo y el trabajo decente son componentes básicos para conseguir una globalización justa y reducir la pobreza. El estado de bienestar social implica entonces el respeto a los derechos que una persona necesita para gozar de una buena calidad de vida, una existencia tranquila, un estado de satisfacción como es tener un empleo digno, un salario justo que satisfaga las necesidades básicas, la vivienda, el acceso a la educación y a la salud.

La importancia del trabajo decente para realizar el desarrollo sostenible ha sido puesto de manifiesto en la Agenda 2030 de Desarrollo Sostenible en el Objetivo 8, y cuya finalidad es: promover el crecimiento económico sostenido, inclusivo y sostenible, el empleo pleno y productivo y el trabajo decente para todos.

Pero la injusticia social es un fenómeno mundial que ha descendido preocupantemente la vida de muchas personas. Esta situación impide el desarrollo pleno de cada individuo y condiciona la forma de vivir de quienes la padecen. En este sentido es evidente la ausencia de un verdadero estado social de derecho como forma de organización política, económica y social encaminada a realizar la justicia social y la dignidad humana mediante el acatamiento de los servidores públicos a los derechos y deberes sociales de orden constitucional, eliminando o disminuyendo los efectos de la desigualdad social de la mayoría de la población. 


\section{Resultados de la reforma laboral}

El propósito inicial de la reforma laboral de dar acceso al mercado laboral a más mexicanos y facilitar la generación de más y mejores empleos formales para atender las necesidades más básicas de los mexicanos, dista mucho de estarse cumpliendo.

En México, como en muchos países latinoamericanos, la pobreza está vinculada con la falta de oportunidades, es decir, muchos ciudadanos no tienen posibilidad de encontrar un trabajo suficientemente bien remunerado. Desde hace diez años, el índice de desempleo se ha desarrollado de manera alarmante, lo que ha provocado también el incremento de la informalidad y la falta de protección social.

Con la crisis económica mundial no se observan posibilidades de generar nuevos empleos, porque además los responsables de las políticas públicas ya no están seguros de cómo crear empleos debido a que la globalización, la innovación tecnológica y la subcontratación están induciendo cambios en la economía mundial, perturbando las estructuras de los mercados laborales nacionales.

De acuerdo con datos arrojados por el Instituto Nacional de Estadística y Geografía (INEGI), durante los meses de enero, febrero y marzo de 2014, la tasa de desocupación de jóvenes de entre 20 y 24 años de edad era de $9.2 \%$, lo cual representa un alto nivel de inactividad.

Una realidad que no se puede ocultar es que actualmente los jóvenes padecen esta crisis de empleo y por ello se ven obligados a crear sus propias fuentes de empleo y de ingresos, que desde luego son informales. La Encuesta Nacional de Ocupación y Empleo 2013, registra que $32.3 \%$ de los jóvenes del país tiene un empleo informal que les permite percibir algún ingreso.

La mayoría de las personas que se incorporan a la economía informal lo hacen porque se encuentran en una situación de necesidad, así que buscan sobrevivir y tener acceso a actividades que les generen ingresos para poder cubrir sus necesidades más básicas. Además, la economía informal se caracteriza por tener un agudo déficit de trabajo decente y un porcentaje desproporcionado de trabajadores pobres.

Algunos datos obtenidos del Informe sobre el desarrollo mundial 2013: Empleo, del Banco Mundial, sostienen que la economía informal se caracteriza por un agudo déficit de trabajo 
decente y un porcentaje desproporcionado de trabajadores pobres. Es cierto que algunas actividades en el rubro de la economía informal pueden brindar condiciones de vida e ingresos razonables, pero la mayoría de las personas que trabajan en este tipo de economía están expuestas a condiciones de trabajo inadecuadas e inseguras y tienen altos niveles de analfabetismo, poca calificación y escasas oportunidades de formación; los ingresos que obtienen no son regulares ni seguros, trabajan más horas y no gozan de acceso a la seguridad social.

El crecimiento desmedido de la economía informal ha propiciado:

a. La expansión de puestos de trabajo precarios y de muy baja productividad.

b. Falta de estabilidad laboral.

c. Una fuente importante de precariedad, de pobreza y de niveles muy bajos de productividad.

d. Obstáculos para el desarrollo económico y social del país.

e. Bajos ingresos por concepto de impuestos, situación que limita la capacidad del Estado para cubrir necesidades de justicia social para los ciudadanos.

f. Que se coloque fuera del sistema de seguridad social, permitiendo que un amplio sector de la población que trabaja en estas condiciones quede marginada de la protección social.

De acuerdo con los resultados de la Encuesta Nacional de Ocupación y Empleo, las cifras durante el segundo trimestre de 2015 revelan que los datos "desestacionalizados", tanto la tasa de informalidad laboral como la tasa de ocupación en este sector, registraron un crecimiento de 0.1 puntos porcentuales con respecto a la del trimestre previo; 6 de cada diez personas trabajan sin ningún tipo de seguridad social, de acuerdo con registros del Instituto Nacional de Estadística y Geografía.

Ante esta situación de crecimiento del desempleo en México, es evidente el crecimiento de la economía informal y con ello la generación de actividades al margen de la legalidad. Así, el trabajo decente comprende el bienestar social al que aspiran las personas durante su vida laboral. Significa la oportunidad de acceder a un empleo decente y productivo que genere un ingreso justo, estabilidad en el empleo, protección social para las familias y mejores perspectivas de desarrollo personal e integración social. 
En este contexto, las nuevas formas de contratación laboral en México no han mejorado ni han incrementado los índices de empleo, tal y como se explica en la exposición de motivos de la iniciativa, al afirmar que "más jóvenes y mujeres podrían tener más oportunidades de empleo y acceder a trabajos de medio tiempo".

El derecho a la estabilidad en el empleo también se vulnera ante las nuevas formas de contratación temporal pues se elimina el principio de permanencia, mientras que persiste la materia de trabajo y se condiciona a que las funciones realizadas sean de la misma naturaleza.

En cuanto al salario como un factor que influye en el nivel de vida de los trabajadores se debe considerar primero que existía, y existe aún, una crisis económica a nivel mundial y nacional desde antes de que fuera aprobada la reforma laboral en México (2012), crisis que obviamente ha deteriorado de forma aguda la capacidad adquisitiva del salario.

El salario mínimo, según la legislación internacional y la mexicana, debe ser suficiente para una familia de cuatro personas en promedio y no solo para una. En el apartado VI del Artículo 123, la Constitución Política de los Estados Unidos Mexicanos menciona que los salarios mínimos generales deberán ser suficientes para satisfacer las necesidades normales de un jefe de familia, en el orden material, social y cultural, y para proveer la educación obligatoria de los hijos. Es ampliamente conocido que lo anterior en México no se cumple, a pesar de ser un precepto constitucional.

El salario mínimo en México, como ya se documentó en el apartado anterior, es de los más bajos en América Latina. En una de las ediciones más recientes del Informe Mundial de Salarios, la Organización Internacional de Trabajo (OIT, 2013, p. 42) subrayó que en México el salario mínimo está por debajo de los niveles del mercado, aún para los trabajadores no calificados.

El deterioro salarial es tan evidente que la OCDE ha denunciado que en México se paga el salario mínimo más bajo de entre todos los países de ese organismo. México es también el país de la OCDE que menos gasta en políticas activas de empleo. Medidas efectivas de activación ayudan a conectar a personas desempleadas e inactivas con puestos de trabajo y pueden jugar un rol fundamental en la reducción de la inactividad. Para lograr estos objetivos, las políticas de activación necesitan fortalecer la motivación de buscar trabajo, así como mejorar la empleabilidad de quienes buscan una ocupación y las oportunidades 
disponibles (OCDE, p. 2015). Otro aspecto interesante que planteó en su momento la iniciativa de reforma a la Ley Federal del Trabajo y que hoy es una realidad, es el hecho de establecer un límite a la generación de salarios vencidos para combatir la indebida práctica de prolongar los juicios.

Los principios generales de derecho laboral señalan que las normas de trabajo tienden a conseguir el equilibrio y la justicia social en las relaciones entre trabajadores y patrones, que el trabajo es un derecho y un deber social, que no es un artículo de comercio, y que exige respeto para las libertades y dignidad de quien lo presta.

El trabajo es un bien de todos, que debe estar disponible para todos aquellos que demuestren competencias para realizarlo. La plena ocupación es, por tanto, un objetivo obligado para todo ordenamiento económico orientado a la justicia y al bien común. Una sociedad donde el derecho al trabajo sea anulado o sistemáticamente negado y donde las medidas de política económica no permitan a los trabajadores alcanzar niveles satisfactorios de vida, no puede conseguir su legitimación ética ni alcanzar la justicia social. Quien está desempleado o subempleado padece, en efecto, las consecuencias profundamente negativas que esta condición produce en la personalidad y corre el riesgo de quedar al margen de la sociedad y de convertirse en víctima de la exclusión social.

¿Qué sucede cuando un trabajador es despedido de su fuente de trabajo?

El artículo 48 de la Ley Federal del Trabajo establece al respecto que si en el juicio correspondiente el patrón no demuestra la causa de la rescisión, el trabajador tendrá derecho a que se le paguen los salarios vencidos contados desde la fecha del despido hasta por un periodo máximo de doce meses, en términos de lo estipulado en la última parte del párrafo anterior. Si al término del plazo señalado en el párrafo anterior no ha concluido el procedimiento o no se ha dado cumplimiento al laudo, se pagarán al trabajador los intereses que se generen sobre el importe de quince meses de salario, a razón del $2 \%$ mensual, capitalizable al momento del pago. Lo dispuesto en este párrafo no será aplicable para el pago de otro tipo de indemnizaciones o prestaciones. Cabe mencionar que el pago de salarios caídos tiene un efecto de reparación del daño ocasionado al trabajador, es decir, se paga al trabajador lo que dejó de percibir porque lo despidieron injustificadamente. 
Esta modificación se hizo bajo el argumento de que el procedimiento laboral no se agotaba (ni se agota, pues en algunas juntas locales la lentitud en el procedimiento sigue siendo una constante) de manera rápida y su desarrollo podía tardar hasta seis u ocho años, de ahí que los juicios laborales resultaran tan largos y que cuando condenaban al patrón demandado al pago de los salarios caídos, este tenía que cubrir el pago de todos los años de salarios que tardaba el juicio, ocasionándole un impacto económico que incluso podía llevarlo a la quiebra.

Aunque la reforma contempla medidas de apremio para quienes notoriamente dilaten el procedimiento a través de la promoción de recursos, diligencias, incidentes y ofrecimiento de pruebas —incluso si el retraso lo ocasiona la autoridad-, el procedimiento laboral en las juntas de conciliación y arbitraje sigue siendo lento.

Los propósitos expuestos durante la exposición de motivos de la reforma laboral de evitar, por un lado, el impacto económico a los patrones que son demandados por sus trabajadores por despido injustificado y que después son condenados en el laudo al pago de salarios caídos y, por el otro, procurar la disminución de los tiempos procesales de los juicios tramitados ante las juntas de conciliación y arbitraje, no se cumplen. Las autoridades laborales reconocen que han sido rebasadas con respecto a la carga de trabajo y que los recursos humanos son insuficientes para enfrentar y resolver en menor tiempo las demandas que cada día se acumulan en sus escritorios.

Esta situación puede desanimar a los trabajadores de reclamar sus derechos o hacer que ante la urgente necesidad de los mismos de contar con recursos económicos para subsistir, se vean obligados a aceptar arreglos económicos ilegales, afectando el derecho de los trabajadores a gozar de justicia social y eliminando el carácter social del derecho laboral.

La realidad laboral en el México actual es que muchos ciudadanos viven un escenario lacerante, alejado de la justicia social y provocado en gran medida por el desempleo, condiciones de trabajo inseguras e inestables, salarios bajos, discriminación, inobservancia de las normas laborales, todo lo cual invalida cualquier posibilidad de disponer de lo necesario para tener una vida digna y que consecuentemente genera estrés entre la clase trabajadora porque las exigencias de su entorno laboral superan su capacidad de hacerles frente o mantenerlas bajo control. 


\section{La relación entre el desempleo y el estrés}

El concepto estrés deriva del inglés stress, que se refiere a "tensión provocada por situaciones agobiantes que originan reacciones psicosomáticas o trastornos psicológicos a veces graves" (Real Academia Española, 2014, p. 976). Otra definición refiere al estrés como: "situación de un individuo, o de alguno de sus órganos o aparatos que por exigir de ellos un rendimiento superior al normal los pone en riesgo de enfermar" (Canda, M. F. et al., 2002, pp. 112 y 113). En la obra especializada sobre el tema se afirma que el estrés:

Es una respuesta personal y el resultado negativo de un desequilibrio entre la presión y la capacidad de la persona para afrontarla. El estrés es la manera como la gente se siente cuando tiene demasiada presión: tiene un nudo en el estómago, tensión en los hombros, incapacidad para dormir, problemas de conducta y así sucesivamente. Estos son los signos físicos, mentales y sociales de que ya no se es capaz de afrontar la situación (Williams, E. y Cooper, L. 2002, p. 6).

El estrés relacionado con el trabajo es un patrón de reacciones que ocurren cuando los trabajadores enfrentan exigencias ocupacionales que no corresponden a su conocimiento, destrezas o habilidades y que los retan a hacer frente a la situación. Cuando existe un desequilibrio entre las exigencias ocupacionales y los recursos del entorno o personales con los que cuenta el trabajador, entonces se presenta una serie de reacciones que pueden incluir: respuestas fisiológicas (aumento del ritmo cardiaco o de presión arterial, hiperventilación, así como secreción de hormonas del “estrés", tales como adrenalina y cortisol); respuestas emocionales (por ejemplo, sentirse nervioso o irritado); respuestas cognitivas (por ejemplo, reducción o limitación de la atención y memoria) y reacciones conductuales (por ejemplo, agresividad, conductas impulsivas, cometer errores) (Houtman, I., Jettinghof, K. y Cedillo, L., 2008, p. 10).

\section{Consecuencias del estrés.}

Los efectos del estrés en niveles muy bajos son positivos ya que son mecanismos que impulsan la acción; en niveles altos son negativos, siendo los más comunes: 
a. Conductuales: alcoholismo, tendencia a sufrir accidentes, adicciones y comportamiento impulsivo.

b. Subjetivos: agresividad, depresión, fatiga, frustración.

c. Cognoscitivos: falta de concentración, deficiente toma de decisiones, bloqueo mental.

d. Psicológicos: hipertensión, taquicardia, enfermedades psicosomáticas.

e. Organizacionales: ausentismo, baja productividad, fallas y errores, insatisfacción y falta de compromiso (Münch, L., 2005, p. 124).

El estrés sentido y expresado en sus diferentes modalidades representa un conjunto de estados y rasgos de la personalidad que disminuyen, inhiben o bloquean las potencialidades de los trabajadores para que se desempeñen con eficiencia y eficacia en su ambiente laboral, ya que tales vivencias afectan la salud mental y el bienestar del personal; entendiendo por salud mental:

La capacidad de proyectar, crear, producir, convivir con el medio y sus semejantes, resolver sus necesidades básicas, cultivar la amistad y promover la paz, generar empatía, amarse, amar y ser amado, superar sus fracasos; pero, sobre todo, disfrutar de sus éxitos, divertirse, ser feliz y compartir esa felicidad con sus familiares y amistades (Antúnez, S.T., 2010, p. 4).

El trabajador que tiene a salvo sus derechos, que goza de seguridad en el empleo, que obtiene los ingresos económicos para cubrir las necesidades básicas de su familia, que goza de buena salud física y mental, o sea con niveles bajos de estrés o sin él, llegará puntual a su lugar de trabajo, se conducirá con optimismo durante su jornada laboral, se desempeñará eficientemente y con responsabilidad, estará coadyuvando al cumplimiento de los objetivos estratégicos de la empresa, que se reflejarán en la productividad, y garantizará que la misma tenga estabilidad, crecimiento y continuidad. 


\section{Conclusión}

El análisis de las reformas laborales en México y su relación con la justicia social, permite arribar a las siguientes conclusiones:

No ha sido evidente que la reforma laboral esté favoreciendo el crecimiento económico del país; por el contrario, existe más desigualdad y vulnerabilidad entre la población de bajos ingresos. Tampoco se ha demostrado una generación de más y nuevos empleos; por el contrario, estos han disminuido y los que existen son eventuales y de baja remuneración, lo que impide a los trabajadores cubrir sus necesidades básicas y aspirar a vivir con dignidad. La tendencia es que aumente la economía informal con nula seguridad social. Con el aumento de la economía informal y el subempleo, el acceso a la seguridad social no está garantizado.

La reforma a la Ley Federal del Trabajo en México está generando un retroceso de las conquistas logradas a partir de las luchas de los movimientos sociales porque ha disminuido los parámetros de respeto y la observancia de los derechos humanos laborales.

Los bajos o insuficientes ingresos de los trabajadores obstaculizan su acceso a los servicios básicos de salud física y mental; asimismo, el desempleo genera tensiones difíciles de afrontar, desembocando en estrés negativo que puede complicar la estabilidad mental y emocional, signo característico de los trastornos mentales asociados tanto con las malas condiciones de trabajo como con el desempleo. Aunado a esto último está la amenaza del desánimo, la subvaloración, la baja autoestima, el aislamiento y, en casos extremos incluso la depresión. 


\section{Bibliografía}

Antúnez, S.T. (2010). La salud mental con Aguirre. Propuesta de Programa de

Gobierno. Documento en archivo electrónico.

Antúnez, S. T. (2014). Ensayo: la salud mental y la calidad de vida. Documento en archivo electrónico.

Avante, J. R. (2013). Apuntes para conocer la Reforma a la Ley Federal del Trabajo [en línea], Secretaría del Trabajo y Previsión Social, México, consulta: 2/06/2016, disponible en: http://ordenjuridico.gob.mx/Publicaciones/Libros2013/laboral-2013web.pdf

Banco Mundial. Informe sobre el desarrollo mundial 2013: Empleo. http://siteresources.worldbank.org/EXTNWDR2013/Resources/82580241320950747192/8260293-1322665883147/Overview_Spanish.pdf

Canda, M. F. et al. (2002). Diccionario de Pedagogía y Psicología, Madrid, Editorial Cultural.

De la Garza, T. E. (Coord.) (2012). La situación del trabajo en México: el trabajo en la crisis, México, Plaza y Valdés.

Evolución del Mercado Laboral en México, Paris, Recuperado de: http://www.keepeek.com/Digital-Asset-Management/oecd/employment/oecdemployment-outlook-2015_empl_outlook-2015-en\#page4

Houtman, I., Jettinghoff, K. y Cedillo, L. (2008). Sensibilizando sobre el estrés laboral en los países en desarrollo. Un riesgo moderno en un ambiente de trabajo tradicional. Consejos para empleadores y representantes de los trabajadores, Organización Mundial de la Salud. Documento en archivo electrónico.

Ley Federal del Trabajo. Diario Oficial de la Federación, noviembre 30 de 2012.

Münch, L. (2005). Administración de capital humano. La gestión del activo más valioso de la organización, primera edición, México, Editorial Trillas.

Organización Internacional del Trabajo (2016). Perspectivas sociales y del empleo en el mundo. Tendencias 2016, Ginebra, OIT.

Real Academia Española (2014). Diccionario de la lengua española, vigésimotercera edición -edición del tricentenario-, México, editorial ESPASA. 
Indicadores de ocupación y empleo 2016, Recuperado de: http://www3.inegi.org.mx/sistemas/temas/default.aspx?s=est\&c=25433\&t=1

Instituto Nacional de Estadística y Geografía. Indicadores de ocupación y empleo 2016, (INEGI) http://www3.inegi.org.mx/sistemas/temas/default.aspx?s=est\&c=25433\&t=1

Instituto Nacional de Estadística y Geografía, Resultados de la Encuesta Nacional de Ocupación y Empleo (cifras durante el primer trimestre de 2015) en http://www.inegi.org.mx/.

Williams, E. y Cooper, L. (2004). Manejo del estrés en el trabajo. Plan detallado para profesionales, México, Editorial El Manual Moderno. 